\title{
Light Induced Frustration of Etching in Fe doped $\mathrm{LiNbO}_{3}$
}

\author{
Ian E Barry and Robert W Eason \\ Optoelectronics Research Centre \\ University of Southampton \\ Southampton, \\ SO17 1BJ \\ U.K. \\ Gary Cook \\ Defence Evaluation Research Agency \\ Malvern \\ WR14 3PS \\ U.K.
}

\begin{abstract}
We report frustration of normal etching in iron doped lithium niobate by incident $488 \mathrm{~nm}$ light. At intensities $>1 \mathrm{Wcm}^{-2}$ etching is fully suppressed. We report the techniques' resolution and the effect of applied electric fields.
\end{abstract}




\title{
Light Induced Frustration of Etching in Fe doped $\mathrm{LiNbO}_{3}$
}

\author{
Ian E Barry and Robert W Eason \\ Optoelectronics Research Centre, University of Southampton, Southampton, SO17 IBJ, U.K. \\ ib(a)orc.soton.ac.uk, rwe (a) orc.soton.ac.uk
}

Gary Cook

Defence Evaluation Research Agency, Malvern, WR14 3PS, U.K. garyc $(0$ taz.dra.hmg.gb

There is considerable interest in etching, structuring, and patterning a wide range of insulators, dielectrics, and semiconductors, for microelectronic and microphotonic device applications. The parameters of most importance for such processes are factors such as etch speed and quality, aspect ratios obtainable, and the degree of complexity associated with details such as lithographic patterning that may be a prerequisite for a process such as electrochemical etching. Techniques may be classified into those that require the use of incident ions, electrons, or photons, such as ion-beam, (including reactive etching), electron-beam or ablative etching, and those that involve wet-etching, which may be a direct electrochemical process, with spatial definition achieved using patterning techniques, or photoelectrochemical etching, which has the added advantage of using spatially patternable light to mediate and control the etching process.

Photoelectrochemical and photoassisted wet-etching has been the subject of numerous recent publications, often involving technologically important optoelectronic materials such as GaN. in both bulk, and thin film formats [1-3]. In all cases, the material is immersed in a cell, surrounded by an etchant such as $\mathrm{KOH}, \mathrm{HCl}$, or $\mathrm{HF}$, and exposed to combinations of applied electric field, and light. Preferentially enhanced etching of the material occurs in those areas exposed to light, at intensities that can be quite modest (of order $\mathrm{mW} \mathrm{cm}$ to a few $\mathrm{W} \mathrm{cm}^{-2}$ ). Additional aspects such as etch anisotropy and dopant-selective etching, are also important, and add further versatility [4].

In all reports of photoelectrochemical etching we have seen however, the action of light is to enhance or assist the etch process. In this paper, we discuss the opposite effect that we have observed in $\mathrm{LiNbO}_{3}$ doped with 0.2 molar \% Fe. On exposure to light at $488 \mathrm{~nm}$, etching of the $-\mathrm{z}$ face is suppressed. Material outside the illuminated area is etched at the normal room

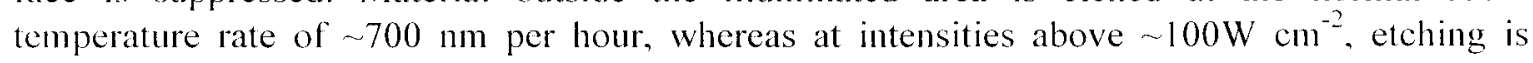
completely suppressed. This light induced frustrated etching (LIFE) behaviour is novel to our knowledge, and arises due to the light induced carrier generation within the Fe doped material.

l'igure 1 is a micrograph of the exposed area, overlayed with calculated power densities incident on the crystal, determined from the known spot size. There are three regions which are easily distinguished here. The central area, labelled $\mathrm{A}$, corresponds to a region which has experienced no etching. Outside this, there exists a threshold area, B, where etching has been reduced, or partially frustrated. The power density at the boundary between these two regions is of order $1 \mathrm{~W}$ $\mathrm{cm}^{-2}$. Beyond this again, in region $\mathrm{C}$, etching proceeds as normal over the rest of the crystal face. 
Light Induced Frustration of Etching in Lithium Niobate, I.E. Barry, Page 2

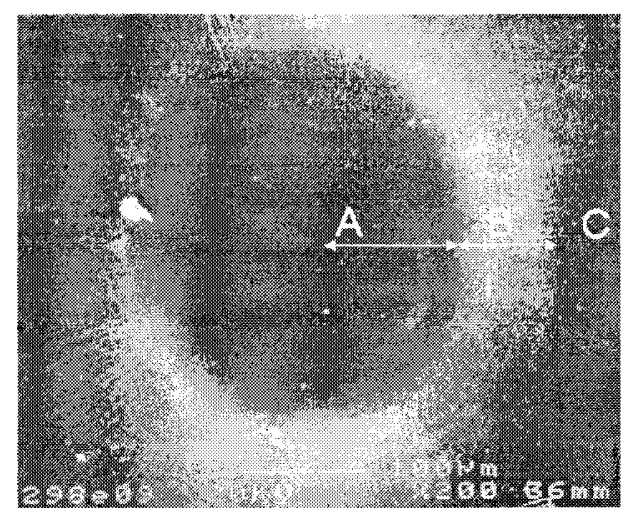

Figure 1: Micrograph of etch-frustrated spot

Figure 2, which is a surface profile trace across the $\mathrm{LiNbO}_{3}$, confirms these results. The frustrated area is shown as a vertical feature of height $\sim 700 \mathrm{~nm}$.

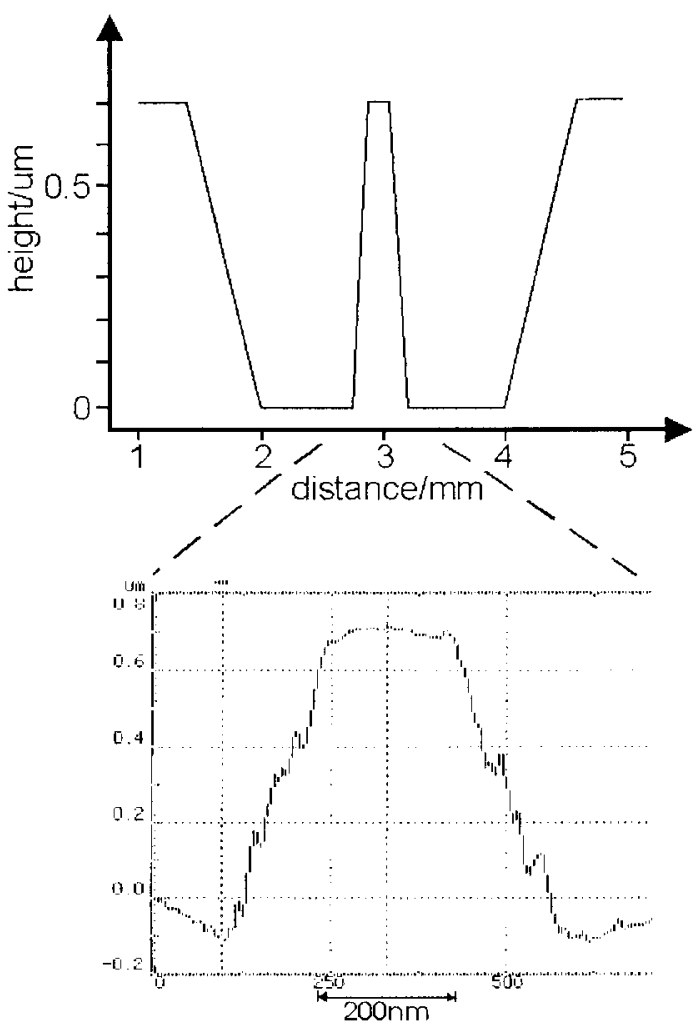

Figure 2: Profilometer trace and schematic of etch-frustrated spot

In conclusion we report a method of optical control of etching in Lithium Niobate. The effect of applied electric fields and the resolution of the process are discussed. 